\title{
Porcine growth hormone induces the nuclear localization of porcine growth hormone receptor in vivo
}

\author{
Hainan Lan ${ }^{1, a, *}$, Huilin Liu ${ }^{2, a}$, Pan Hong ${ }^{1}$, Ruonan Li ${ }^{1}$, and Xin Zheng ${ }^{1, *}$
}

\author{
* Corresponding Authors: \\ Hainan Lan \\ Tel: +86-0431-84517235, Fax: +86-0431-84517235, \\ E-mail: tougao@jlau.edu.cn \\ Xin Zheng \\ Tel: +86-0431-84517235, Fax: +86-0431-84517235, \\ E-mail: zhengtougao@163.com
}

'College of Animal Science and Technology Jilin Agricultural University, Changchun 130118, China

${ }^{2}$ College of life sciences, Jilin University, Changchun

130118, China

a Hainan Lan and Huilin Liu have contributed equally.

ORCID

Hainan Lan

https://orcid.org/0000-0002-2248-8292

Xin Zheng

https://orcid.org/0000-0002-7073-7329

Submitted Aug 10, 2017; Revised Sept 16, 2017; Accepted Sept 21, 2017
Objective: Recent studies have challenged the traditional paradigm that growth hormone receptor (GHR) displays physiological functions only in the cell membrane. It has been demonstrated that GHR localizes to the cell nucleus and still exhibits important physiological roles. The phenomenon of nuclear localization of growth hormone $(\mathrm{GH})$-induced GHR has previously been described in vitro. However, until recently, whether GH could induce nuclear localization of GHR in vivo was unclear.

Methods: In the present study, we used pig as an animal model, and porcine growth hormone (pGH) or saline was injected into the inferior vena cava. We subsequently observed the localization of porcine growth hormone receptor (pGHR) using multiple techniques, including, immunoprecipitation and Western-blotting, indirect immunofluorescence assay and electronmicroscopy.

Results: The results showed that pGH could induce nuclear localization of pGHR. Taken together, the results of the present study provided the first demonstration that pGHR was translocated to cell nuclei under pGH stimulation in vivo.

Conclusion: Nuclear localization of pGHR induced by the in vivo pGH treatment suggests new functions and/or novel roles of nuclear pGHR, which deserve further study.

Keywords: Porcine Growth Hormone; Porcine Growth Hormone Receptor; Nuclear Translocation; In vivo

\section{INTRODUCTION}

Growth hormone (GH) processes extensive physiological functions [1]. According to statistics, GH has more than 300 functions and roles [2]. GH initiates intracellular signalling by interacting with growth hormone receptor (GHR) localized at the cell membrane. GH binding to GHR induces phosphorylation of the Janus kinase (JAK2), which subsequently triggers a series of intracellular signalling proteins, including signal transducer and activator of transcription (STAT) as well as extracellular regulated protein kinases, and these signalling proteins contribute to all of the actions of GH [1].

The previous paradigm of the action mechanism of GH/GHR is that after GH binds to the membrane-GHR, JAK2, and GHR are activated, and subsequently, down-stream signalling molecules are recruited and activated. These signal molecules transport from the cytoplasm to the nucleus, where they regulate target gene transcription. However, twenty years ago, the phenomenon of GH and/or GHR nuclear localization was reported in vitro [3]. However, until recently, researchers had determined the functions of this protein in nuclei. Water et al showed that GHR nuclear localization is associated with the proliferative status of cells and tissues [4]. Subsequently, several studies have reported the new functions of nuclear-GHR [5-6]. Indeed, nuclear translocation of cell membrane GHR can be divided 
into three basic processes: GHR internalization, cytoplasmic transport and nuclear localization. Strous et al have indicated that the ubiquitin system is required for GHR internalization. Furthermore, it has been reported that importin $\alpha$ and $\beta$ (IMP $\alpha / \beta)$ are responsible for GHR nuclear translocation [4].

Porcine GH possesses extensive biological activities in pigs, e.g., improving the feed efficiency, increasing the growth rate, and changing the carcass composition [7-9]. It has been demonstrated that the porcine liver is an important target of porcine growth hormone (pGH), which endogenously expresses $\mathrm{pGHR}$, and studies have demonstrated that porcine hepatocytes are an important model for studying the interactions between $\mathrm{pGH}$ and $\mathrm{pGHR}$.

However, thus far, there is no report of the nuclear localization of pGHR in domestic animals in vivo. In addition, it remains unclear whether $\mathrm{pGH}$ could induce nuclear localization of pGHR in vivo, although the phenomenon of GHinduced GHR nuclear localization has been reported in vitro. There are many differences in vitro and in vivo, and the in vivo environments are more complicated compared to the in vitro environments. In the present study, we selected the pig as an animal model to study pGHR nuclear translocation under pGH treatment in vivo. We demonstrated that the in vivo treatment of pGH could induce nuclear localization of pGHR.

\section{MATERIALS AND METHODS}

\section{Antibodies and reagents}

pGH was purchased from Sigma-Aldrich (St. Louis, MO, USA). Anti-pGHR antibody was obtained from Abcam (Cambridge, England). Nuclear/Cytoplasmic Extraction Reagent Kits were obtained from Pierce (Rockford, IL, USA). Bovine serum albumin (BSA), non-fat milk, tissue lysis buffer and enhanced chemiluminescence (ECL) were purchased from Beyotime (Shanghai, China). Glutaraldehyde and paraformaldehyde were obtained from Hua-Yi Biotechnology (Changchun, China). Polyvinylidene fluoride (PVDF) membranes were obtained from Millipore (Bedford, MA, USA). The colloidal goldconjugated secondary antibody was purchased from Abcam (England). Second antibodies were purchased from Kai-Ji Biotechnology (Nanjing, China).

\section{pGH treatment in vivo}

Pigs (Landrace, average $\sim 15 \mathrm{~kg}$ body weights) were used following experimental treatment, and the study was approved by the Animal Ethical Committee of Jilin Agricultural University. The animals were housed three per cage at room temperature. Pigs were randomly assigned to experimental and control groups, and the experimental group was treated with pGH $(0.5 \mathrm{mg} / \mathrm{kg})$. The $\mathrm{pGH}$ dose that was used was the same as that from a previous study, and the control group was treated with saline. The pigs were deeply anaesthetized using pentobarbital ( $100 \mathrm{mg} / \mathrm{kg})$. The abdominal cavity was opened, the inferior vena cava was exposed, and pGH was injected into the inferior vena cava. After $30 \mathrm{~min}$, the livers were isolated, and subsequent experiments were performed as described below.

\section{Immunoelectron microscopy}

After the pGH treatment described above, the left liver lobes were isolated. The liver samples (size $1 \mathrm{~mm}^{3}$ ) were subsequently sectioned. The samples were fixed with $4 \%$ glutaraldehyde for $2 \mathrm{~h}$ at $4^{\circ} \mathrm{C}$. After washing, the tissue samples were post-fixed in $2 \%$ osmic acid solution for $3 \mathrm{~h}$ at $4^{\circ} \mathrm{C}$, dehydrated in graded ethanol solutions and further infiltrated and embedded in araldite. Ultrathin sections ( 40 to $50 \mathrm{~nm}$ ) of tissue samples were cut using a Leica Ultracut UCT ultramicrotome (Leica Company, Solms, Gemany). After washing three times, the sections were incubated with $5 \%$ normal goat serum for 30 min to block non-specific binding. The sections were subsequently rinsed and incubated with the primary antibody (antiGHR antibody) for $1 \mathrm{~h}$. After washing, the sections were incubated with a secondary antibody (goat anti-mouse) conjugated to 10-nm colloidal gold particle for $30 \mathrm{~min}$, followed by three washes. After staining with uranyl acetate for $10 \mathrm{~min}$, the sections were examined using transmission electron microscopy (EM) (HITACHI H-7650, Hitachi Limited, Tokyo, Japan).

\section{Immunoprecipitation and Western-blotting}

After pGH treatment, the liver tissues were sampled. The Nuclear/Cytoplasmic Extraction Reagent Kit (Pierce, USA) was used to isolate nuclear and cytosol fractions of the liver tissue according to the manufacturer's instructions. For subsequent immunoprecipitation, the cytosolic or nuclear fractions were incubated with the anti-pGHR antibody or an irrelevant isotype-matched antibody overnight at $4^{\circ} \mathrm{C}$. The immunoprecipitated proteins were subsequently subjected to sodium dodecyl sulfate-polyacrylamide gel electrophoresis, followed by immunoblotting with anti-pGHR antibodies as previously described [10].

\section{Indirect immunofluorescence assay}

The indirect immunofluorescence assay (IFA) experimetns were carried out according our previous procedure [11]. In breif, after pGH injections, the porcine hepatocytes were rapdliy isolated and fixed with $4 \%$ paraformaldehyde for $20 \mathrm{~min}$, then permeabilized for $5 \mathrm{~min}$ in $0.5 \%$ Triton X-100. After washing with phosphate-buffered saline (PBS), the cells were then treated with $2 \%$ BSA for $1 \mathrm{~h}$, after which, the porcine hepatycytes were incubated with anti-pGHR or isotype control monoclonal antibody (negative control) for $1 \mathrm{~h}$. The cells were washed again with PBS and incubated with fluorescein isothiocyanate-labelled goat anti-mouse immunoglobulin $\mathrm{G}$ for 
$1 \mathrm{~h}$ in the dark. In addiion, the propidium iodide was used to stain the cell nucleus. After finan three wahses, the cells were analysed using confocal laser scanning microscopy (Olympus FV1000, Olympus, Tokyo, Japan).

\section{Statistical analysis}

The data are presented as the mean values \pm standard error of triplicate samples.

\section{RESULTS}

\section{Charicterization of pGHR expression}

We first established pGHR expression in porcine liver tissue (Landrace, $\sim 15 \mathrm{~kg}$ ) by western-blot using an anti-pGHR antibody and, as indicated in Figure 1, a pGHR band ( 120 KDa) was detected in liver tissue, and the control antibody showed no effects (Figure 1).

\section{GHR's localization with or without pGH stimulation}

After the pGH injections, as described in the Materials and Methods section, the cytoplasmic and nuclear fractions from the liver tissue were isolated using the Nuclear/Cytoplasmic Extraction Reagent Kit (Pierce, USA). The isolated Nuclear/ Cytoplasmic proteins were subjected to immunoprecipitation and Western-blot experiments. As shown in Figure2A, after pGH treatment, pGHR was detected in the nuclear extract and was also detected in the cytoplasm. pGHR from liver tissue after saline treatment was detected in the cytoplasm but not in the nucleus.

Next, IFA assays were used to further confirm these observations. As illustrated in Figure 2B and 2C, after pGH treatment, pGHR was primarily localized in the cytoplasm and

\section{Control Anti-pGHR}

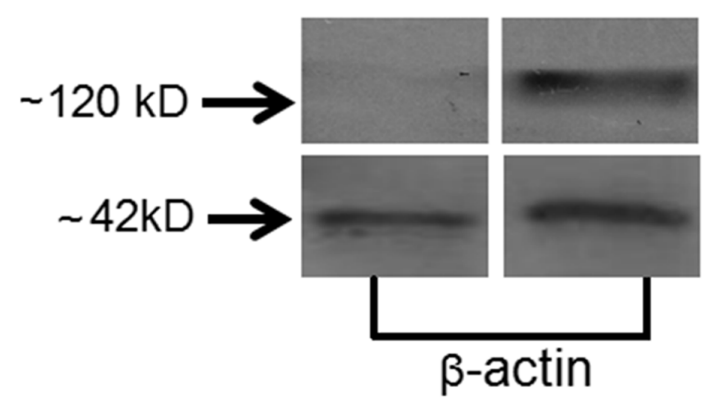

Figure 1. Western-blot analysis of pGHR (porcine growth hormone receptor) expression in porcine liver tissue. The liver tissue samples were collected, and subsequently, the extracted protein was subjected to sodium dodecyl sulfatepolyacrylamide gel electrophoresis and transferred to polyvinylidene fluoride membranes. The membranes were incubated with 3\% bovine serum albumin to block non-specific binding. After washing, the membranes were incubated with primary antibody (anti-pGHR), followed by a secondary antibody, for $1 \mathrm{~h}$ each. Enhanced chemiluminescence ( $E C L$ ) was used to detect the immune-reactive bands. nucleus. By contrast, pGHR in liver tissue after saline treatment was primarily localized in the cytoplasm, and little or no pGHR signalling was detected in the cell nucleus. By the co-localization analysis of Confocal laser scanning microscope (yellow signal), it can be found that $\mathrm{pGH}$ and $\mathrm{pGHR}$ may form a dimer in the cytoplasm and nuclei (Figure 2D). In this section, we did not performed immunohistochemistry experiments to detect and localize pGHR, this is because that the anti-pGHR antibody applied in the current experiments is not suitable to be used in the immunohistochemistry assay.

In addition, we also performed EM experiments to observe pGHR nuclear localization, and as shown in Figure 2E (see indicated arrows), pGHR was detected in cell nuclei and the nuclear membrane.

\section{pGHR's nuclear localization is pGH-depanent}

To determine whether pGHR nuclear localization is specifically induced by pGH, a pGHR-specific antibody and control antibody (B32 developed in a previous study and demonstrated to compete with pGH for GHR binding [11]) were pre-injected in the inferior vena cava for 30 min prior to $\mathrm{pGH}$ injection. As indicated in Figure 3, pGHR was not detected in the cell nucleus in liver tissue pre-injected with B32. To further evaluate if the pGHR's nuclear localization is GH-depandent, we also performed the corresponding experiments by the injection of human growth hormone (hGH) or bovine growth hormone (bGH), we found that hGH and bGH also could induce pGHR's nuclear localization. These observations are similar with that of the in vitro experiments. These findings suggest that the nuclear localization of pGHR may be GHdependent.

\section{DISCUSSION}

In the present study, to our knowledge, we demonstrated the first exploration of pGHR nuclear translocation induced by pGH in vivo in domestic animals. The results showed that pGHR was primarily localized to the cytoplasm without $\mathrm{pGH}$ stimulation. By contrast, pGHR was strongly localized to cell nuclei after pGH treatment, suggesting a new scientific study point, namely, the potential functions of nuclear- pGHR in porcine liver tissue, which deserve further study.

pGH plays important roles in the growth and development of pigs [12]. Almost all tissues express pGHR, suggesting that pGH has potential effects on all tissues. The traditional paradigm of the GH/GHR interaction is that after GH binding to membrane-GHR, JAK2 and GHR are activated though phosphorylation, followed by activation of down-stream signalling molecules [13]. These signal molecules transport from the cytoplasm to the nucleus, where they regulate target gene transcription. However, a number of studies have demonstrated the phenomenon of GHR nuclear localization, although the 
A.
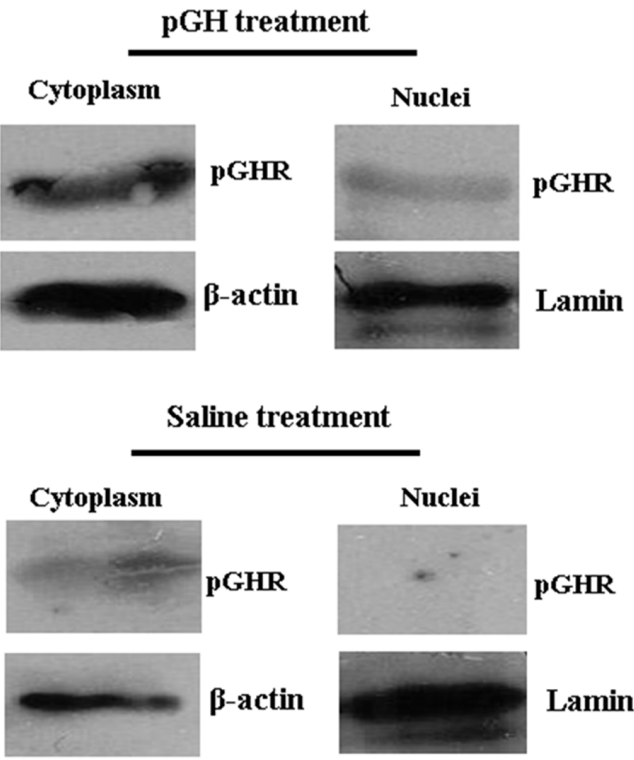

C.

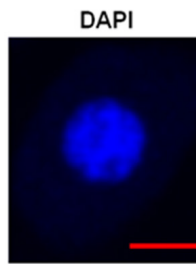

pGHR
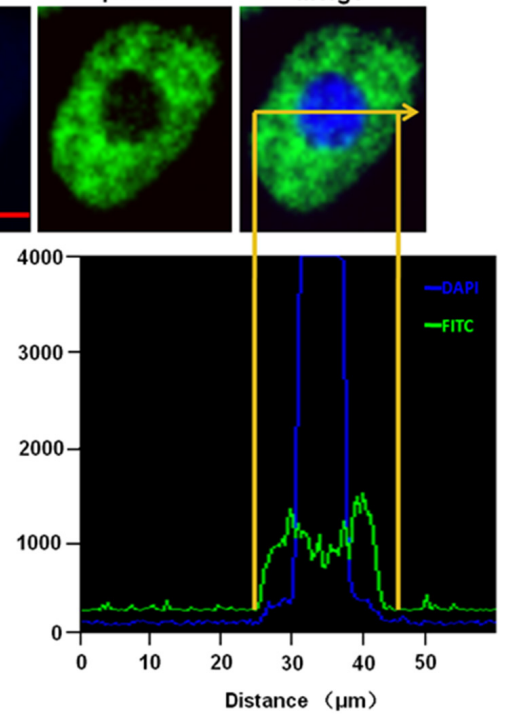

\section{E. pGHR}

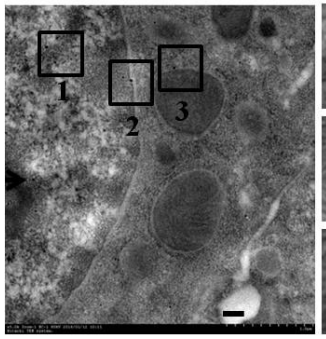

Inset

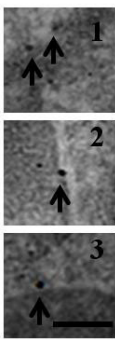

Control

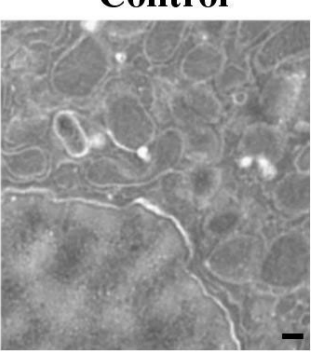

B.

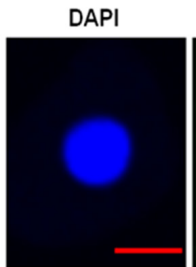

pGHR
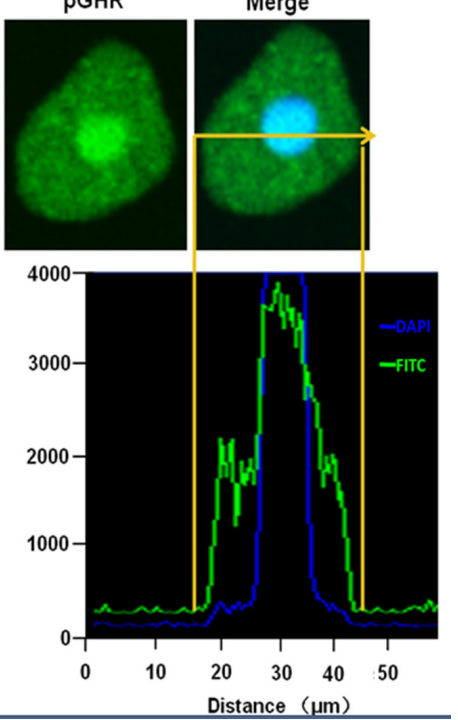

D. DAPI

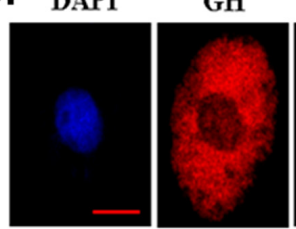

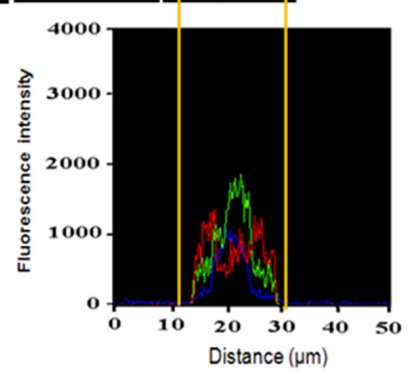

Figure 2. (A) Analysis of porcine growth hormone receptor (pGHR) nuclear localization by Western-blotting. The Nuclear/Cytoplasmic Extraction Reagent Kit (Pierce, Rockford, IL, USA) was used to isolate nuclear and cytosol fractions of the liver tissue. Subsequently, for immunoprecipitation, the cytosolic or nuclear fractions were incubated with the anti-pGHR antibody or an irrelevant isotype-matched antibody overnight at $4^{\circ} \mathrm{C}$. The immunoprecipitated proteins were subsequently subjected to sodium dodecyl sulfatepolyacrylamide gel electrophoresis, followed by immunoblotting with anti-pGHR antibodies. (B), (C) Analysis of pGHR localization by immunofluorescence assay (IFA). After porcine growth hormone $(\mathrm{pGH})$ or saline treatment, the liver tissue samples were collected. The following IFA experiments were performed as described as in the Materials and Methods section. The corresponding histograms show the co-localization of pGHR (green signal) and the cell nuclei (blue signal), which were achived by scanning across the one cell. (D) The co-localization analysis of GH and pGHR. (E) Immunoelectron microscopy of pGHR's nuclear localization. After pGH treatment, liver tissues were collected. Ultrathin sections were prepared and treated as described in the Materials and Methods section. The sections were examined using transmission electron microscopy (HITACHI H-7650, Tokyo, Japan). Arrow points to colloidal gold particles. N, nuclei; C, cytoplasm. Bar: 200 nM. The figure represents three independent experiments. 


\section{B32 hGH bGH}

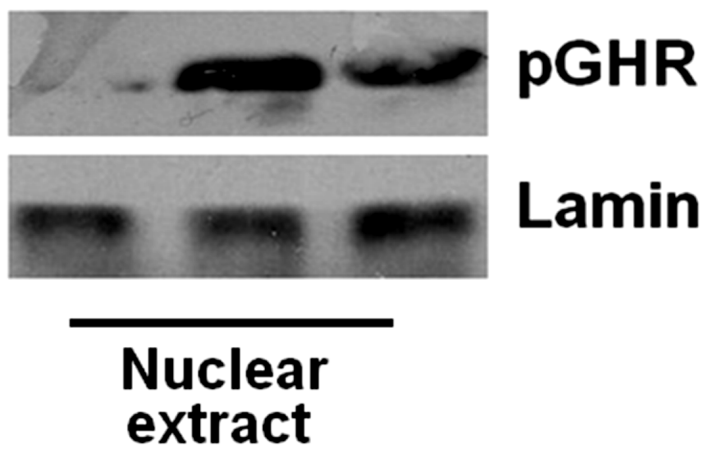

Figure 3. Analysis of porcine growth hormone receptor (pGHR) nuclear localization by Western-blotting. After treatments with the indicated ligands, the Nuclear Extraction Reagent Kit (Pierce, Rockford, IL, USA) was used to isolate nuclear fractions of the liver tissue. Subsequently, the nuclear fractions were subsequently subjected to sodium dodecyl sulfate-polyacrylamide gel electrophoresis, followed by immunoblotting with anti-pGHR antibodies.

specific and exact functions of nuclear-GHR in cell nuclei remain unclear. Walters et al showed that nuclear-GHR associated with cell proliferation and that abnormal nuclear localization of GHR may induce tumour generation [4]. In the present study, we observed the phenomenon of the nuclear localization of pGHR induced by $\mathrm{pGH}$ in vivo. However, the functions of nuclear-localized pGHR remain unclear and deserve further study.

Indeed, the mechanism(s) of pGHR nuclear translocation remains to be fully understood. In a previous study, we showed that IMP $\alpha / \beta$ is involved in $\mathrm{pGHR}$ nuclear translocation in porcine hepatocytes [6]. In general, although nuclear translocation of cell membrane proteins can be divided into three basic steps, including protein internalization, cytoplasmic transport and nuclear localization, nuclear translocation is a complicated molecular procedure involving many molecules and cellular organelles. For example, epidermal growth factor receptor (EGFR) nuclear translocation is involved in many cytoplasmic molecules and organelles, involving multiple nuclear localization pathways for EGFR [14-17]. Currently, studies on the mechanism(s) of GHR nuclear transport remain limited, and these studies were only performed in vitro. The present study establishes a model for the study of GHR nuclear localization in vivo.

Conway-Campbell et al showed that GHR prolongs STAT5 activation based on its nuclear localization. In addition, Graichen et al showed that nuclear-growth hormone binding protein (GHBP) enhances STAT5-mediated transcription [18]. However, until recently, the molecular mechanisms through which GHBP and GHR display these physiological activities remain unclear. In addition, we cannot exclude that, similar to EGFR, nuclear-GHR can be used as a transcription factor.

Taken together, the results of the present study provide the first evidence that pGH can induce pGHR nuclear translocation in vivo, indicating that pGHR not only functions in the cell membrane but also in cell nuclei.

\section{CONFLICT OF INTEREST}

We certify that there is no conflict of interest with any financial organization regarding the material discussed in the manuscript.

\section{ACKNOWLEDGMENTS}

This work was supported by the National Natural Science Foundation-Young investigator grant program (Grant number 31602022). This project was partially supported by the Scientific Research projects of the Thirteenth Five-Year plan of Jilin Province Department of Education (Grant number 2016178).

\section{REFERENCES}

1. Brooks AJ, Waters MJ. The growth hormone receptor: mechanism of activation and clinical implications. Nat Rev Endocrinol 2010;6:515-25.

2. Waters MJ, Hoang HN, Fairlie DP, et al. New insights into growth hormone action. J Mol Endocrinol 2006;36:1-7.

3. Lobie PE, Mertani H, Morel G, et al. Receptor-mediated nuclear translocation of growth hormone. J Biol Chem 1994;269: 21330-9.

4. Conway-Campbell BL, Wooh JW, Brooks AJ, et al. Nuclear targeting of the growth hormone receptor results in dysregulation of cell proliferation and tumorigenesis. Proc Natl Acad Sci USA 2007;104:13331-6.

5. Conway-Campbell BL, Brooks AJ, Robinson PJ, et al. The extracellular domain of the growth hormone receptor interacts with coactivator activator to promote cell proliferation. Mol Endocrinol 2008;22:2190-202.

6. Lan HN, Hong P, Li RN, et al. Growth hormone (GH)-specific induction of the nuclear localization of porcine growth hormone receptor (pGHR) in the porcine hepatocytes. Domest Anim Endocrinol 2017;61:39-47.

7. Chung CS, Etherton TD, Wiggins JP. Stimulation of swine growth by porcine growth hormone. J Anim Sci 1985;60:11830.

8. Etherton TD, Wiggins JP, Evock CM, et al. Stimulation of pig growth performance by porcine growth hormone: determination of the dose-response relationship. J Anim Sci 1987;64: 433-43.

9. Figueiredo MA, Boyle RT, Sandrini JZ, et al. High level of GHR nuclear translocation in skeletal muscle of a hyperplasic transgenic zebrafish. J Mol Endocrinol 2016;56:47-54.

10. Lan HN, Li W, Fu ZL, et al. Differential intracellular signalling properties of the growth hormone receptor induced by the 
activation of an anti-GHR antibody. Mol Cell Endocrinol 2014; 390:54-64.

11. Lan HN, Jiang HL, Li W, et al. Development and characterization of a novel anti-idiotypic monoclonal antibody to growth hormone, which can mimic physiological functions of growth hormone in primary porcine hepatocytes. Asian-Australas J Anim Sci 2015;28:573-83.

12. Campbell RG, Steele NC, Caperna TJ, et al. Interrelationships between energy intake and endogenous porcine growth hormone administration on the performance, body composition and protein and energy metabolism of growing pigs weighing 25 to 55 kilograms live weight. J Anim Sci 1988;66:1643-55.

13. Brown RJ, Adams JJ, Pelekanos RA, et al. Model for growth hormone receptor activation based on subunit rotation within a receptor dimer. Nat Struct Mol Biol 2005;12:814-21.

14. Du Y, Shen J, Hsu JL, et al. Syntaxin 6-mediated Golgi translocation plays an important role in nuclear functions of EGFR through microtubule-dependent trafficking. Oncogene 2014; 33:756-70.

15. Lin SY, Makino K, Xia W, et al. Nuclear localization of EGF receptor and its potential new role as a transcription factor. Nat Cell Biol 2001;3:802-8.

16. Lo HW, Ali-Seyed M, Wu Y, et al. Nuclear-cytoplasmic transport of EGFR involves receptor endocytosis, importin $\beta 1$ and CRM1. J Cell Biochem 2006;98:1570-83.

17. Wang YN, Wang H, Yamaguchi H, et al. COPI-mediated retrograde trafficking from the Golgi to the ER regulates EGFR nuclear transport. Biochem BiophysRes Commun 2010;399: 498-504.

18. Graichen R, Sandstedt JEL, Goh OG, Törnell J, Lobie PE. The growth hormone-binding protein is a location-dependent cytokine receptor transcriptional enhancer. J Biol Chem 2003; 278:6346-54. 